\title{
Aprendizaje y transferencia de conocimiento para la política pública del sector aeronáutico colombiano $^{1}$
}

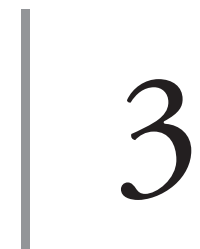

https://doi.org/10.21830/9789585380226.03

Alejandra Cerón R.
Yuber Rico V.
Oscar I. Parra
Aérea Colombiana

Escuela de Postgrados de la Fuerza Aérea Colombiana

Resumen. El sector aeronáutico ha adquirido una relevancia fundamental en el desarrollo de la seguridad humana y multidimensional, de manera que es prioritario encontrar la relación de estos avances políticos a través del aprendizaje y de la transferencia del conocimiento desde modelos foráneos que imponen las tendencias internacionales del mercado y de la tecnología. Al observar las dinámicas internacionales y locales alrededor de los temas aeronáuticos, se encuentra que existe confluencia entre la seguridad nacional y otras concepciones, en medio de una transferencia de conocimientos y prácticas, que han sido adaptadas en un proceso de aprendizaje que necesariamente relaciona a los sujetos con su ambiente. Si bien se proyecta la renovación del sector aeronáutico acorde con las necesidades locales y las demandas internacionales, se requieren esfuerzos posteriores, políticos y científicos.

Palabras clave: aprendizaje; industria aeroespacial; política pública; seguridad humana; transferencia de conocimientos.

1 Este capítulo forma parte de los resultados del Grupo Gisic, de la Escuela de Postgrados de la Fuerza Aérea Colombiana. Los puntos de vista y los resultados presentados en este capítulo pertenecen a los autores y no reflejan necesariamente los de las instituciones participantes.

2 Doctora en Estudios Políticos y Relaciones Internacionales de la Universidad Nacional de Colombia. Magíster en Gestión de Organizaciones de la Université du Québec à Chicoutimi (UQAC) de Canadá. Socióloga de la Universidad Nacional de Colombia. Orcid: https://orcid.org/0000-0002-56427949 - Contacto: luz.ceron@epfac.edu.co

3 Sociólogo e internacionalista de la Pontificia Universidad Javeriana. Estudiante de la Maestría en Estudios Políticos Latinoamericanos de la Universidad Nacional de Colombia. Orcid: https://orcid. org/0000-0003-3026-7964 - Contacto: oparrac@unal.edu.co 


\section{Introducción}

En la globalización y en las nuevas aproximaciones a la seguridad se aprecia la importancia del sector aeronáutico y se puede evidenciar en sus proyecciones. Las industrias relacionadas con el sector tienen un crecimiento estimado de $\$ 15$ trillones de dólares, además de contar con 20.000 proveedores. En Colombia, el sector crece entre el $6 \%$ y el $7 \%$ entre aviación comercial, de carga, fabricación de autopartes y reparación de aviones (Delgado, 2018). En la actualidad, se han formulado diversos planes para el desarrollo del sector desde diferentes agencias y posturas institucionales:

- Plan Estratégico para el Desarrollo del Sector Aeronáutico y Aeroespacial 2018-2022, creado por la Fuerza Aérea, el Ministerio de Defensa y la Asociación Colombiana de Productores Aeroespaciales (ACOPAER).

- Plan Estratégico Aeronáutico 2030, producto del Foro Sector Aéreo 2030, de la Aeronáutica Civil.

- Plan de Navegación Aérea para Colombia, liderado también por la Aeronáutica Civil y compilado en tres tomos entre 2015 y 2017.

En este escenario, es necesario plantear los siguientes interrogantes: ¿Cuál ha sido el aprendizaje del sector sobre las políticas públicas? ¿Cuál es el origen del aprendizaje político sobre el potencial del sector aeronáutico para la seguridad?, ¿acaso es foráneo o parte de una necesidad reflexionada? Además de que el desarrollo y las proyecciones del sector aeronáutico no están pensadas para el caso colombiano y sus dinámicas de seguridad, se encuentra que los modelos y las reglamentaciones internacionales provienen de extrapolaciones del sistema comercial liberal, aunque pretenden incluir dentro de su agenda exploraciones sobre la sostenibilidad ambiental, la ciberseguridad y la reducción de la desigualdad de condiciones entre países (Liu, 2017, pp. 4-5). Estas estrategias sugieren homogeneización de medidas y protocolos, a la vez que emplean la reglamentación como vehículo de optimización de los procesos operacionales y del negocio. 
Este capítulo está organizado de la siguiente manera: primero, se expone la metodología postempirista e interpretativa como forma de aproximación empírica al tema y a las fuentes documentales; segundo, se realiza una breve introducción al aprendizaje y la transferencia de conocimiento en las políticas públicas y un cuestionamiento desde la posibilidad de la autonomía; tercero, se describe cómo se desarrolla la transferencia de conocimiento y el aprendizaje en el sector aeronáutico y su relación con la seguridad; cuarto y último, se proponen unas breves conclusiones con respecto al estado de los planes de acción de las organizaciones nacionales de la aeronáutica en relación con los discursos predominantes de las organizaciones internacionales y las posibilidades de aprendizaje en la transferencia de conocimiento.

\section{Métodos}

Las teorías y metodologías postempiristas acuñan aspectos esenciales de la realidad social que caracterizan a la interacción intersubjetiva más allá de los rasgos materiales y del objeto observable: lo discursivo y lingüístico como intermediación humana por antonomasia entre el sujeto y la realidad. Hay una contradicción fundamental con los métodos cuantitativos que pretenden sostener las explicaciones de los fenómenos sociales desde la distancia experimental, mientras que los métodos postempiristas responden a una aproximación más sensible de los valores y de las ideas de los sujetos, donde radica el poder explicativo de las contiendas políticas y de los procesos sociales (Cerón, 2019, p. 35).

Las perspectivas contemporáneas del análisis político, entre las que se destacan la propuesta elaborada por Frank Fischer (2003), plantean un cambio en los criterios epistémicos de la concepción acerca de la política, abandonando la visión de los análisis tradicionales de costos y beneficios del proceso de toma de decisiones. En cambio, las propuestas postempiristas logran reivindicar el carácter social y de larga duración que implican los procesos políticos. Desde esta orientación se reconoce la importancia que tiene en la formación de los procesos políticos la participación de diversos grupos sociales en la generación de los discursos y las ideas sociales que acompañen a los procesos. 
Este artículo utiliza un método interpretativo, en el análisis discursivo y de organización de la comunidad involucrada con el sector aeronáutico, con el propósito de extraer los valores y los principios organizacionales que proyectan los actores. Esta profundización alrededor de las ideas que participan en el cambio político involucra una transferencia de conocimiento y una gradualidad del aprendizaje. Esto implica reconocer una necesaria relación entre la política, como dirección de un proyecto político, con el conocimiento científico y tecnológico; la posibilidad del aprendizaje como actividad del sujeto en la que utiliza las herramientas adquiridas en sus condiciones de existencia.

La interpretación requiere encontrar sentido a la experiencia del otro, la cual se encuentra relatada en un documento recuperado por un autor. La lectura de este relato traduce y concreta el entendimiento del escritor; una doble construcción, del lector y del escritor. En el caso del lector, este eleva y selecciona lo que en su criterio es lo más importante o relevante del texto, especialmente en lo que concierne a la ideología y escuela teórica (Denzin, 2009, pp. 88-90). En el estudio de las políticas públicas, la estrategia interpretativa debe estar orientada hacia la revisión de la retórica, la argumentación y las narrativas empleadas por los autores de las políticas públicas, de los discursos y de los documentos oficiales (Ginger, 2006, p. 335). La pregunta ¿qué se pretende con esta premisa o conclusión? es fundamental para comprender la intencionalidad, el orden de prioridades y las formas de organización. Específicamente, en este escrito se desea encontrar la manera como el desarrollo de la aeronáutica se ha vuelto fundamental para la seguridad de Colombia. Según este planteamiento, eso solo es posible a través de la profundización de los discursos nacionales e internacionales; si hay consenso, réplica o adaptación de los cambios de la política.

\section{Resultados}

En este acápite es necesario resaltar la manera como se desarrollan los procesos de transferencia de políticas y cómo estos se relacionan en particular con el tema del conocimiento y la tecnología, a partir del establecimiento de modelos de crecimiento y desarrollo a nivel mundial. Este planteamiento 
se enfoca sobre todo en el establecimiento de políticas a través de recomendaciones externas o acuerdos foráneos para aplicar medidas convenientes a intereses internacionales. Con el mismo realismo con el que se ejecuta este razonamiento, también se arguye sobre las ceñidas posibilidades de autorrealización: ¿Existe realmente una proyección o iniciativa propia?

Según Dussauge, la transferencia se entiende como "un proceso autónomo, voluntario, en el que el aprendizaje transnacional por parte de los hacedores de políticas desempeńa un papel fundamental" (Dussauge, 2012, p. 55). Con esta visión se plantea la posibilidad de que en la transferencia de política se pueda iniciar la búsqueda de nuevas ideas, programas, políticas e instituciones participantes que en suma defenderían la pretensión de la innovación. Es decir, para que suceda la transferencia de conocimiento se requiere de una nula resistencia en la recepción de la información, de la tecnología o de la cooperación. Ahora, lo que se debería cuestionar es la intencionalidad de esta transferencia, la motivación de la fuente a incluir al otro dentro de estos desarrollos.

En este sentido, después de transferir el conocimiento se debe revisar de qué manera se importa al lugar de recepción. Es en este punto donde se cuestiona la gradualidad del aprendizaje, dependiendo de cuáles elementos del conocimiento transferido se deja intacto. Al respecto, Rose (2005) propone una escala de aprendizaje de lecciones que varía entre la transferencia absoluta y el aprendizaje activo: photocopying - copying - adaptation - hybrid - synthesis - disciplined inspiration - selective imitation (Rose, 2005, p. 81). Pero, ¿qué factores determinan que un proceso se ubique en algún lugar de esta escala?

La respuesta probablemente se encuentre en la motivación para aprender, en la necesidad y en la coerción. En 1969, Fernando Henrique Cardoso y Enzo Faletto publicaron Dependencia y desarrollo en América Latina, un ensayo que plasma un análisis del capitalismo global, de las relaciones entre las potencias económicas con los países denominados "subdesarrollados" o de "tercer mundo”. Cardoso y Faletto (1977) encuentran además relaciones de dependencia entre los países de centro con los de la periferia: estos tienen un precario desarrollo industrial y riqueza en recursos naturales, mientras que aquellos 
poseen fortaleza tecnológica y de capital. Las relaciones de dependencia son resultado de las relaciones internacionales de producción entre las élites de los países periféricos con los capitalistas del centro, que permiten la explotación de los recursos y de la mano de obra barata de la periferia. Ahora, el direccionamiento del desarrollo parecería ser unidireccional, es decir, de la periferia hacia el centro: el centro requiere de la periferia para sostener su desarrollo, y la periferia necesita del centro para subsistir. Si se habla desde la teoría de la dependencia en relación con la transferencia de conocimientos, ¿realmente el aprendizaje tiene autonomía o sucede forzadamente?

Cuando se valora el paso de la seguridad nacional a la seguridad humana o a la seguridad multidimensional, se debe incluir en el análisis los cambios históricos y las reflexiones que evocan en los académicos y en los tomadores de decisiones. Estas transformaciones son constantes y las interacciones entre los actores estatales y los no estatales son dinámicas y requieren de un aprendizaje para confrontar las nuevas relaciones. De este lugar de reflexión y aprendizaje surge la Teoría de la Dependencia planteada en América Latina, como respuesta académica a los modelos económicos extranjeros y como resultado autónomo de observar el funcionamiento del capitalismo global desde el subdesarrollo. Las condiciones materiales imponen desafíos particulares para cada autoridad estatal y para cada fuerza militar; los conflictos políticos y sociales que ha padecido Colombia desembocan en respuestas culturales y políticas autóctonas, y del contraste con modelos internacionalmente válidos. Ningún país del mundo ha tenido la experiencia multifacética del conflicto colombiano, lo cual permite, a su vez, que las respuestas y las capacidades del Estado colombiano sean únicas.

La historia condena a los sujetos vehementemente a la realidad presente, así como le permite al sujeto convertirse en parte activa del proceso histórico. En consecuencia, las definiciones de la seguridad son resultado de los efectos de los eventos y de la reflexión histórica. De esta forma, las interpretaciones en torno a la seguridad tienen la necesidad de desplazarse junto con los eventos históricos, las discusiones filosóficas y políticas. Por tanto, hoy la dependencia económica y tecnológica son temas ineludibles de seguridad. 


\section{Discusión}

La comparación de los textos normativos internacionales con la política aeronáutica colombiana evidencia la búsqueda por conseguir la autonomía de la política pública. Se entiende que la política también es un proceso de construcción que toma como referencias modelos comprensivos de la realidad y de la experiencia. Este ejercicio comparativo busca establecer el nivel de dependencia de la política pública colombiana, en el aprendizaje del sector aeronáutico, en relación con los avances normativos de las organizaciones internacionales y con las demandas del mercado. Al mismo tiempo, esta maniobra es un móvil para profundizar la discusión sobre la seguridad multidimensional y la centralidad del sector aeronáutico sobre los procesos de globalización.

La importancia del sector aeronáutico para la seguridad multidimensional se debe al creciente papel del conocimiento y de la tecnología en las sociedades, tanto en las formas de consumo como en las relaciones sociales. Evidentemente, unos actores se encuentran en desventaja y en dependencia porque no poseen las capacidades económicas o el conocimiento para producir autónomamente los medios necesarios para mantener la regularidad de sus interacciones; podría llamarse normalidad o idoneidad. El sector aeronáutico, en este sentido, es fundamental para el transporte de personas y de mercancías, para conectar diversos grupos humanos y optimizar la producción. Si se considera que el transporte de alcance planetario es un elemento indispensable para los procesos de globalización, es imperativo entonces concebir al sector aeronáutico como fundamento de la seguridad del sistema económico y social actual.

De estas prácticas pueden resultar distintos mecanismos de transferencia del conocimiento, las cuales pueden resumir iniciativas que han sido establecidas a través de directivas o instrucciones, resultado de las rutinas definidas en las interacciones o el establecimiento estratégico de rutinas. Por ejemplo, tres años después de que la Organización de la Aviación Civil Internacional (OACI) celebrara el Convenio de Chicago en 1944, en Colombia se ratificó mediante la Ley 12 de 1947, con lo cual se fijaron en el país los protocolos de comunicación o de emergencias de acuerdo con las normativas internacionales para la gestión de la aviación. 
Sin embargo, es con el Manual de seguridad de la aviación expedido por la OACI que Colombia debe alinear sus políticas de seguridad aeroportuaria con los estándares normativos y procedimentales de la seguridad internacional (Habib, 2018). Consecuentemente, la Aeronáutica Civil desarrolló los Reglamentos Aeronáuticos de Colombia (RAC), con la finalidad de regular los movimientos aeronáuticos en el territorio colombiano. Estos "deberán ser actualizables y flexibles para adaptarlos al entorno económico del sector aeronáutico y al cumplimiento de estándares y tratados internacionales, con el propósito de incentivar aún más la competencia en el mercado nacional” (Díaz, 2016, p. 126).

$\mathrm{Al}$ revisar los objetivos estratégicos de la OACI 2005-2010, se encuentran cinco generales: (1) seguridad operacional, (2) seguridad de la aviación, (3) protección del medioambiente, (4) eficiencia, y (5) continuidad e imperio de la ley. Grosso modo, el argumento transversal de la $\mathrm{OACI}$ es la gestión del riesgo y el mejoramiento de los protocolos y las operaciones a través de la OACI misma, como institución modelo que lidera las agendas de las organizaciones aeronáuticas locales. Específicamente, se trata de estandarizar los procedimientos utilizando mecanismos como las auditorías, la ratificación de los Convenios de Chicago y del derecho aeronáutico internacional, la cooperación técnica, los planes correctivos regionales, la liberalización de los sectores aeronáuticos, entre otros. Uno de los pilares que más llama la atención dentro de la narrativa de la $\mathrm{OACI}$ es el de desarrollo sostenible; de la mitigación de los efectos negativos de la industria sobre el medio ambiente, mientras el crecimiento empresarial sigue intacto. Se incluye la emisión de gases a nivel local y mundial, así como la producción de ruido, y se busca la cooperación con la Convención Marco de las Naciones Unidas sobre el Cambio Climático (OACI, 2004).

En cuanto a los objetivos estratégicos de la OACI para 2011-2013, se encuentra que se mantienen los tres primeros y la generalidad discursiva de los planteamientos. Se incluye la investigación de accidentes aéreos, la cooperación cívico-militar, la identificación de personas, la actualización de datos y estadísticas, los avances sobre estudios meteorológicos, radiofrecuencias y navegación, y la insistencia alrededor de la transparencia. Aquí se hace hincapié sobre dos temas fundamentales: la tecnificación y la seguridad, para aumentar 
la eficiencia de la industria y del comercio, mientras se reducen los riesgos sobre ilícitos (OACI, 2010).

También se debe señalar que la Asociación Internacional del Transporte Aéreo (IATA, 2018), en colaboración con la School of International Futures (SoIf), realizaron un documento para prever el futuro de la industria en 2035. En el centro argumental del texto se encuentra la necesidad de que la industria aeronáutica se vuelva adaptable frente a la incertidumbre de estos tiempos de globalización y cambio tecnológico. De esta forma, se plantean la posibilidad de actuar en múltiples escenarios, entre los cuales se encuentran: la búsqueda de fuentes de energía alternativas, la ciberseguridad, los eventos climáticos, la volatilidad de la economía global, los conflictos geopolíticos, la privacidad y la vigilancia, las enfermedades y las pandemias, el terrorismo y la emisión de gases y de ruido. En breve, las respuestas requieren de la institucionalización, del fortalecimiento de las relaciones con organismos públicos y privados, la inversión en tecnología, infraestructura y energías alternativas, la prevención interagencial de ciberseguridad y terrorismo, el cuidado de los clientes y la búsqueda de mercados emergentes.

En ambas agendas, tanto la OACI como la IATA persiguen la cooperación transnacional para mantener la efectividad del negocio aeronáutico y lidiar con las amenazas contra la seguridad. Sin embargo, parece seguir la ambigüedad sobre la seguridad a través de la institucionalización o de la liberalización. Tal vez se calcula que la homogeneización de las acciones y el respeto por una autoridad supranacional disminuya los riesgos a la seguridad tanto económica como de las personas.

En noviembre de 2009, la OACI realizó una Auditoría de la Seguridad de la Aviación (UsAP), producto de la cual afirmó que en Colombia hay fallas de coordinación entre las agencias de seguridad, y que la Aeronáutica Civil no evalúa los riesgos eficientemente en la totalidad de las terminales aéreas, especialmente por la carencia de suficientes inspectores en relación con la magnitud de movimientos entre los aeropuertos (Habib, 2018, pp. 48-49). Mientras en esta inspección Colombia recibió el 63,3\% de implementación efectiva, en el 2017 había obtenido una calificación del 74,3 \%. En respuesta, como se sugirió en la introducción del escrito, se han formulado tres planes desde diferentes instancias institucionales: 
1. Plan de Navegación Aérea para Colombia, de la Aeronáutica Civil (2017), que consta de tres volúmenes separados por temáticas: (1) requerimientos operacionales, (2) instalaciones y servicios, y (3) regulación. En este plan se contempla una ampliación de las funciones de los operadores aéreos y la optimización de las capacidades tecnológicas con las que cuentan los aviones y los aeropuertos para mejorar la gestión de riesgos. Por ejemplo, se hace énfasis en la Gestión del Tránsito Aéreo mediante la automatización de los sistemas de navegación, el mejoramiento de los mecanismos de comunicación y la obtención de información en tiempo real. Asimismo, se pretende ampliar los estudios en meteorología para elaborar y compartir los datos, para después realizar análisis y pronósticos. También se proyecta un servicio de búsqueda y salvamento, control de fauna y de incendios desde la Planificación de Operaciones de Aeródromo.

2. Plan Estratégico Aeronáutico 2030 (Aeronáutica Civil, 2018), elaborado para el Foro Sector Aéreo 2030, organizado por la Aeronáutica Civil. Este plan se concentra en seis líneas de acción: (1) competencias institucionales, (2) conectividad y competitividad, (3) infraestructura y sostenibilidad ambiental, (4) seguridad operacional y seguridad de la aviación civil, (5) industria aeronáutica y cadena de suministro, y (6) desarrollo del talento humano en el sector. En especial para este plan, se debe resaltar el énfasis en el desarrollo del turismo para el postconflicto y la conexión con las regiones, además de certificaciones de calidad de las autopartes que se producen en el país y del incentivo de la triada empresa-Estado-universidad.

3. Plan Estratégico para el Desarrollo del Sector Aeronáutico y Aeroespacial 2018-2022 (Fuerza Aérea Colombiana, 2018), desarrollado por la Fuerza Aérea Colombiana, el Ministerio de Defensa y la Asociación Colombiana de Productores Aeroespaciales (Acopaer). En este plan, que pretende alinearse con los objetivos de Política de Seguridad y Defensa del Plan Nacional de Desarrollo 2015-2018, se establecen dos objetivos estratégicos: fortalecer el sistema de certi- 
ficación aeronáutico Ecosistema y consolidar el sector aeronáutico fomentando el comercio de bienes y servicios relacionados.

La posible transferencia de conocimiento al sector aeronáutico colombiano puede provenir de convenios internacionales, organizaciones transnacionales y desarrollos científicos globales que configuran normativas y regulaciones del comportamiento de las industrias y de las empresas. Es claro que sucede un proceso de endogeneización de aquella transferencia, en el cual se yuxtaponen las capacidades con los requerimientos. En especial, los planes se desmarcan parcialmente de los lineamientos internacionales, no por fractura o transgresión, sino por el reconocimiento de las necesidades y capacidades propias (autorreferenciación). Con el Plan de Navegación Aérea para Colombia se busca especialización técnica, mientras que en el Plan Estratégico Aeronáutico 2030 y el Plan Estratégico para el Desarrollo del Sector Aeronáutico y Aeroespacial 2018-2022 se proyecta la explotación de las industrias nacionales emergentes, como las autopartes o el turismo.

\section{Conclusiones}

La innovación política en materia aeronáutica es una necesidad sentida para el desarrollo del país. En este sentido, los últimos gobiernos nacionales han reconocido que aún faltan decretos y leyes que dinamicen el sector, específicamente acciones relacionadas con la transferencia tecnológica y del conocimiento. Además, se considera que los impuestos tienen un impacto directo en el desarrollo competitivo de la industria, por lo cual los empresarios aseguran que es oportuno presionar el desarrollo del sector y superar el divorcio que existe entre las universidades y el sector.

Dado que, por su naturaleza, el sector aeronáutico está parametrizado con los intereses internacionales, tiene dimensiones inmensas y, por lo tanto, para comprenderlo es necesario caracterizarlo. Además, el sector es muy regularizado y requiere la integración de muchos actores, entre los cuales tienen especial importancia los operadores aeronáuticos y el gobierno para lograr un rol que agregue valor a la industria. 
No sería justo aplicar los intervalos de aprendizaje parametrizados por Rose (2005) a esta industria, en especial por el estado incipiente de la política sobre el sector aeronáutico. Sin embargo, es relevante afirmar que los planes descritos tienen una fuerte influencia de las agendas de la OACI y de la IATA. Sobre todo, las organizaciones nacionales tienen poca autonomía para fijar planes y acciones debido a la supervisión de los organismos internacionales, a la presencia de capital foráneo y a la coerción de los mercados. Colombia, como país receptor de la tecnología y del conocimiento técnico, no posee capacidad propositiva o de interpelación sobre sus propios aprendizajes porque, bien sea dicho, el aprendizaje viene precisamente de experiencias exitosas de otros países o de las reglamentaciones de organismos multilaterales, donde tampoco tiene mucha influencia. La dependencia tecnológica y económica también genera una dependencia en la transferencia de conocimientos. De hecho, el aprendizaje que se entrevé forma parte del surgimiento de prácticas espontáneas para reducir costos y para aprovechar nuevos emprendimientos.

La dependencia económica y tecnológica se debe considerar como un problema de seguridad multidimensional y, por lo tanto, el aprendizaje como una necesidad de la seguridad, incluso para lograr autonomía en la producción de los conocimientos. El desarrollo del sector aeronáutico se proyecta como una posibilidad para fortalecer la agencia propia frente a los intereses y las relaciones internacionales. Si el aprendizaje depende de los efectos de la injerencia externa, este va a propender a ajustarse a los estándares internacionales, desajustados para las necesidades locales. En consecuencia, la seguridad tiene un inevitable vínculo con la autonomía para sostenerse; la dependencia condiciona a los Estados a una seguridad que no protege completamente a los individuos o a la sociedad, sino que se enfoca esencialmente en favorecer al apoderado.

En cuanto a la contribución de estos planes y aprendizajes a la seguridad, se debe apuntar la importancia de que hayan incluido ampliamente componentes propuestos por la OACI y en la IATA, que el sector aeronáutico debe considerar para contribuir a la seguridad de las personas, como la ciberseguridad y la sostenibilidad ambiental, junto con la ya conocida prevención contra el terrorismo o el tráfico de drogas y demás ilícitos. En Colombia, se ha 
recibido con cierta pasividad la liberalización del sector y la aceptación de la OACI como autoridad legítima del sector, de la cual acepta recomendaciones y exigencias. Por otra parte, después de la mitigación de los riesgos y del aumento de la eficiencia, el país no parece hacer una apuesta profunda por considerar el desarrollo de la aeronáutica como un componente esencial para la seguridad.

\section{Referencias}

Aeronáutica Civil. (2017). Plan de Navegación Aérea para Colombia.

Aeronáutica Civil. (2018). Plan Estratégico Aeronáutico 2030.

Asociación Internacional del Transporte Aéreo [IATA]. (2018). Future of the Airline Industry.

Banco Interamericano de Desarrollo [BID]. (2001). Competitividad: El motor del crecimiento. BID. https://publications.iadb.org/publications/spanish/document/Competitividad-elmotor-del-crecimiento.pdf

Cardoso, F. H., \& Faletto, E. (1977). Dependencia y desarrollo en América Latina: Ensayo de interpretación sociológica [Serie Documentos Teóricos, 1]. Instituto de Estudios Peruanos. https://repositorio.iep.org.pe/bitstream/handle/IEP/1027/Cardoso_Faletto_ Dependencia-desarrollo-Am\%C3\%A9rica-Latina.pdf?sequence=1\&isAllowed=y

Cerón, A. (2019). La política colombiana de ciencia y tecnología: Análisis contemporáneo [Tesis doctoral, Universidad Nacional de Colombia]. Repositorio UNAL. https://repositorio.unal.edu.co/bitstream/handle/unal/75567/52362113\%20-\%202020.pdf?sequence $=1 \&$ is Allowed $=y$

Delgado, P. (2018, septiembre 2). En Colombia hay industria aeronáutica. El Espectador. https:// www.elespectador.com/noticias/economia/en-colombia-hay-industria-aeronautica/

Denzin, N. (2009). Qualitative inquiry under fire: Toward a new paradigm dialogue. Left Coast Press.

Departamento Nacional de Planeación. (2017). Desarrollo productivo del sector aeronáutico.

Díaz, O. (2016). Análisis del desarrollo reciente del transporte aéreo en Colombia. Revista Transporte y Territorio, (14), 122-143. https://doi.org/10.34096/rtt.i14.2432

Dussauge, M. (2012). La transferencia de políticas como fuente de innovación gubernamental: promesas y riesgos. Revista Estado, Gobierno y Gestión Pública, (12), 51-79.

Fischer, F. (2003). Reframing public policy: Discursive politics and deliberative practices. Oxford University Press.

Fuerza Aérea Colombiana. (2018). Plan Estratégico para el Desarrollo del Sector Aeronáutico y Aeroespacial 2018-2022.

Ginger, C. (2006). Interpretive content analysis: Stories and arguments in analytic documents. En D. Yanow \& P. Schwartz-Shea (Eds.), Interpretation and method: Empirical research methods and the interpretive turn (pp. 331-348). M. E. Sharpe. 
52 Volumen I. Panorama en seguridad y defensa visto desde las instituciones de educación superior de las Fuerzas Armadas.

Habib, A. (2018). Eficacia de la seguridad en la aviación civil colombiana: Factor estratégico para la seguridad nacional y el desarrollo del pais [Tesis de maestría, Escuela Superior de Guerra].

Liu, F. (2017). The current challenges of the International Air Transport. La Aviación en Cifras, 3-7.

Organización de Aviación Civil Internacional [OACI]. (2004). Objetivos estratégicos de la OACI para 2005-2010. https://www.icao.int/Documents/strategic-objectives/strategic_ objectives_2005_2010_es.pdf

Organización de Aviación Civil Internacional [OACI]. (2010). Objetivos estratégicos de la OACI para 2011-2013.

Rose, R. (2005). Learning from comparative public policy: A practical guide. Routledge. 